\title{
Bacteriology of Sputum Samples: A Descriptive Cross-sectional Study in a Tertiary Care Hospital
}

\author{
Bijendra Raj Raghubanshi, ${ }^{1}$ Bal Man Singh Karki ${ }^{1}$ \\ 'Department of Microbiology, KIST Medical College and Teaching Hospital, Gwarko, Lalitpur.
}

\section{ABSTRACT}

Introduction: Lower respiratory tract infection is a common infection and accounts for a greater burden of disease worldwide. It is a great challenge to the clinician and still more, with increasing antimicrobial resistance. Its empirical treatment may vary according to the type of causative organisms. The objective of this study is to identify the pathogenic microorganisms and their antimicrobial susceptibility pattern from sputum sample.

Methods: This descriptive cross-sectional study was conducted in KIST Medical College and Teaching Hospital from February 2015 to January 2016. Ethical approval was taken from institutional review committee prior to the study with reference no. 0051/2014/15. Data on culture and sensitivity of isolates from sputum samples were collected from the records of the hospital. Sample collection, processing, identification of microorganisms and antimicrobial susceptibility tests were performed according to the Clinical and Laboratory Standards Institute guidelines. All the data were tabulated in an Excel sheet and analyzed using SPSS version 20.

Results: Out of 2318 samples, 694 (29.93\%) sputum samples at 95\% confidence interval (737.21650.79) were reported as culture positive. Klebsiella was the most common isolate followed by Pseudomonas, Escherichia coli, Acinetobacter, Staphylococcus aureus, Candida albicans, Streptococcus pneumoniae, Streptococcus pyogenes, and others. Imipenem and vancomycin showed the most sensitivity towards gram-negative and gram-positive bacteria respectively.

Conclusions: Proper diagnosis, identification of causative agents and their antimicrobial susceptibility pattern are important steps to limit the irrational use of antimicrobials. Prescribing antimicrobials empirically in the case of suspected lower respiratory tract infection is difficult.

Keywords: antimicrobial drug resistance; culture; respiratory tract infections; sputum culture.

\section{INTRODUCTION}

Lower respiratory tract infections (LRTIs) are common infections in the general population, however, older individuals and patients with chronic diseases or compromised immune function are prone to these infections. LRTIs account for a greater burden of disease worldwide than ischaemic heart disease, cancer, malaria or human immunodeficiency virus infection so managing patients with LRTIs is a great challenge to a clinician. ${ }^{1}$
Different societies like the British Thoracic Society (BTS) and Infectious Disease Society of America (IDSA) have formulated the main guidelines on managing LRTIs. ${ }^{2,3}$ The identification of pathogenic organisms causing LRTIs is very important for treatment. ${ }^{3}$ The most common

Correspondence: Dr. Bijendra Raj Raghubanshi, Department of Microbiology, KIST Medical College and Teaching Hospital, Imadol, Lalitpur. Email: raghu2_47@yahoo.co.in, Phone: +977-9841332403. 
pathogens isolated from sputum samples are Klebsiella pneumoniae(39.5\%) followed by Pseudomonas(25\%), Escherichia coli $(11.5 \%)$, Staphylococci $(11.5 \%)$ and others (3.8\%). ${ }^{4}$ In comparison to infections caused by susceptible bacteria, increased morbidity and mortality are associated with infections caused by resistant bacteria. ${ }^{5,6}$ Infections caused by resistant bacteria led to prolonged hospital stays, increased health care costs and in many cases untreatable infections. ${ }^{7}$

Thus, this study was conducted to identify the pathogenic microorganisms and their antimicrobial susceptibility pattern from sputum sample.

\section{METHODS}

This descriptive cross-sectional study was done at the Department of Microbiology of KIST Medical College and Teaching Hospital. Ethical approval was taken from the institutional review committee of KISTMCTH prior to the study with reference no. 0051/2014/15. It was conducted from February 2015 to January 2016. Laboratory records from Clinical laboratory services of KIST Medical College and Teaching Hospital were reviewed for the period of 2011 July to 2015 June. All the sputum samples submitted for culture and sensitivity from patients presenting in outpatient departments and inpatient departments who were suspected of having LRTIs were included in the study. Contaminants growths were excluded.

Convenient sampling was done and the sample size was calculated using the formula,

$$
\begin{aligned}
\mathrm{n} & =\mathrm{Z}^{2} \times(\mathrm{p} \times \mathrm{q}) / \mathrm{e}^{2} \\
& =(1.96)^{2} \times 0.5 \times(1-0.5) /(0.03)^{2} \\
& =0.9604 / 0.0009 \\
& =1067.11 \\
& =1068
\end{aligned}
$$

where,

$\mathrm{n}=$ required sample size

$p=$ prevalence of bacterial isolation $(50 \%)$

$q=1-p$

$\mathrm{e}=$ margin of error, $3 \%$

$Z=1.96$ at $95 \% \mathrm{Cl}$

The calculated minimum sample size was 1068. As convenient sampling was done, the sample size was doubled to 2136 , however, the total sample taken was 2318.
In routine clinical laboratory services processing, samples were cultured in five percent blood agar and MacConkey agar and chocolate agar. Inoculation was done with the help of a nichrome wire loop. Blood and MacConkey agar were incubated aerobically at $37^{\circ} \mathrm{C}$ for 48 hours. Chocolate agar was incubated at $37^{\circ} \mathrm{C}$ in a candle jar. The growth of mixed organisms without any predominant growth of a particular organism was reported as normal flora. The sputum samples yielding significant pathogenic organisms were reported as positive cultures. Bacterial identification was done by colony morphology, gram staining, and standard biochemical tests. ${ }^{8}$ Antimicrobial susceptibility test was performed by Kirby-Bauer disc diffusion technique. Different antimicrobial panels were used for different groups of microorganisms and second-line antimicrobials were used only when necessary following the Clinical and Laboratory Standards Institute (CLSI) guidelines. ${ }^{9}$ All the data were tabulated in an Excel sheet and analyzed using SPSS version 20 .

\section{RESULTS}

A total of 2318 sputum culture samples were included for analysis. Out of 2318 samples, 694 (29.93\%) sputum samples were reported at $95 \%$ confidence interval (737.21-650.79\%) as culture positive. Out of the 2318 sputum culture reports analyzed, significant pathogens were not isolated in 1624 samples. Multiple pathogens were obtained from 15 samples.

More than 18 different types of microorganisms were isolated. The most common organism isolated from the sputum sample in this study was Klebsiella species $176(24.82 \%)$. Subsequently other common organisms were Pseudomonas 139 (19.6\%), Escherichia coli 110 $(15.5 \%)$, Acinetobacter 66 (9.3\%), Staphylococcus aureus 63 (8.88\%), Candida albicans 48 (6.77\%), Streptococcus pneumonia 23 (3.24\%), Streptococcus pyogenes $20(2.82 \%)$ and others non-albicans (Candida, Citrobacter, Enterococcus, Enterobacter, Proteus, Haemophilus, Nocardia, etc).

Klebsiella was found to be the most common microorganism in all age groups while other microorganisms also followed a similar pattern (Table 1). 


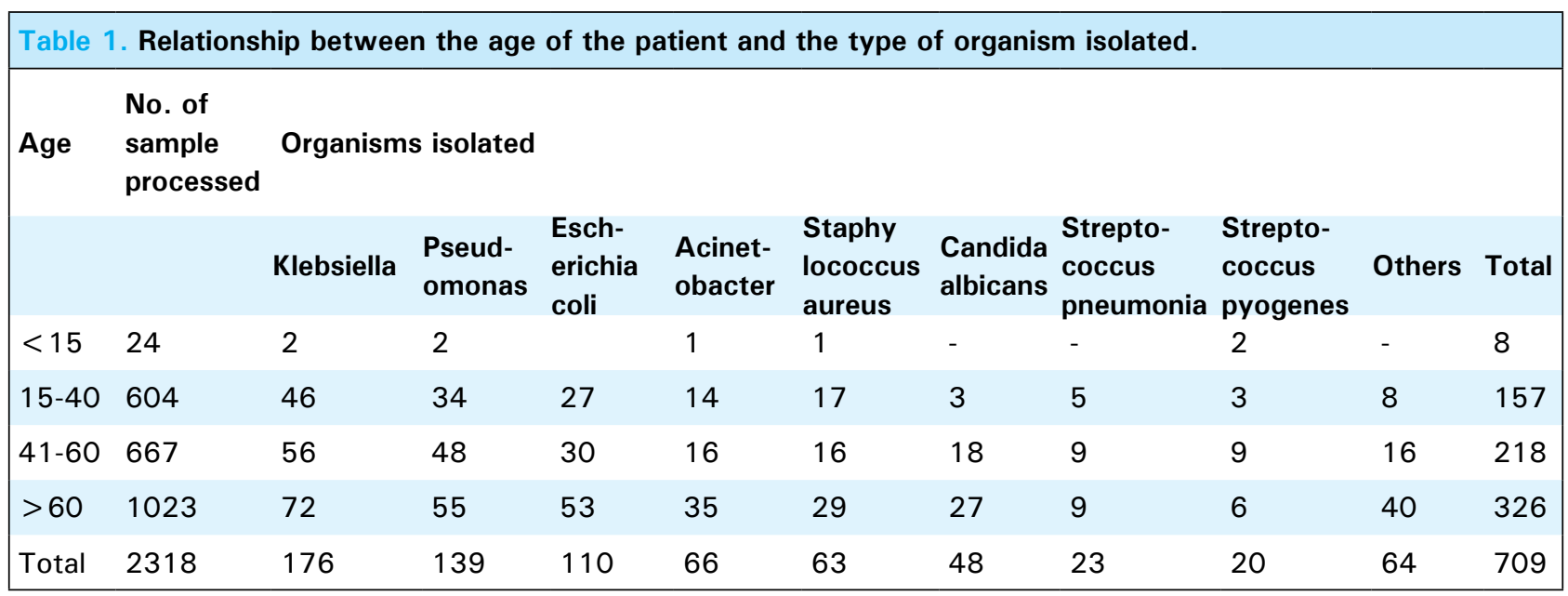

None of the antimicrobials had $100 \%$ efficacy except vancomycin. However, it was used for gram-positive cocci only. For gram-negative organisms, imipenem had the maximum efficacy of $84.33 \%$ against all isolates and $86.9 \%$ against the most common organism (Table
2). Out of tested isolates, most of the organisms were found to be sensitive to Amikacin (79.66\%), followed by Nitrofurantoin (76.66\%) and Gentamicin (70.49\%) whereas Ampicillin and Nalidixic acid had efficacy of $16.66 \%$ and $22.22 \%$ only.

\begin{tabular}{|c|c|c|c|c|}
\hline Antimicrobials & $\begin{array}{l}\text { No. of isolates } \\
\text { tested }(\%)\end{array}$ & $\begin{array}{l}\text { No. of sensitive } \\
\text { isolates }(\%)\end{array}$ & $\begin{array}{l}\text { No. of intermediate } \\
\text { isolates }(\%)\end{array}$ & $\begin{array}{l}\text { No. of resistant } \\
\text { isolates }(\%)\end{array}$ \\
\hline Amikacin & $288(100 \%)$ & 207 (71.87) & - & $81(28.12)$ \\
\hline Ampicillin & 409 (100\%) & $61(14.91)$ & $4(0.97)$ & $344(84.1)$ \\
\hline AMC & $224(100 \%)$ & $16(7.15)$ & - & $208(92.85)$ \\
\hline Carbenicillin & $92(100 \%)$ & $64(69.56)$ & $2(2.17)$ & $26(28.26)$ \\
\hline Cefazolin & $331(100 \%)$ & $52(15.7)$ & $6(1.81)$ & $273(82.47)$ \\
\hline Cefepime & $173(100 \%)$ & $27(15.6 \%)$ & - & 146 (84.39) \\
\hline Cephotaxime & $346(100 \%)$ & $158(45.66)$ & $10(2.89)$ & $178(51.44)$ \\
\hline Ceftriaxone & $219(100 \%)$ & $124(56.62)$ & $1(0.45)$ & $94(42.92)$ \\
\hline Ceftazidime & $105(100 \%)$ & $68(64.76)$ & $1(0.95)$ & $36(34.28)$ \\
\hline Ciprofloxacin & $573(100 \%)$ & $371(64.74)$ & $5(0.87)$ & $197(34.38)$ \\
\hline Cotrimoxazole & $394(100 \%)$ & $202(51.26)$ & - & $192(48.73)$ \\
\hline Eryhromycin & $100(100 \%)$ & $62(62)$ & $2(2)$ & $36(36)$ \\
\hline Gentamycin & $596(100 \%)$ & $423(70.97)$ & $11(1.84)$ & $162(27.18)$ \\
\hline Imipenum & $83(100 \%)$ & 70 (84.33) & $2(2.4)$ & $11(13.25)$ \\
\hline Meropenum & $153(100 \%)$ & $100(65.35)$ & $3(1.96)$ & $50(32.67)$ \\
\hline Ofloxacin & $236(100 \%)$ & $93(39.4)$ & $1(0.42)$ & $142(60.16)$ \\
\hline Tobramycin & $110(100 \%)$ & $93(84.54)$ & $1(0.9)$ & $16(14.54)$ \\
\hline Piparacillin & $88(100 \%)$ & $49(55.68)$ & $1(1.13)$ & $38(43.18)$ \\
\hline Vancomycin & $65(100 \%)$ & $65(100)$ & - & - \\
\hline
\end{tabular}




\section{DISCUSSION}

This study was conducted to find out the pattern of microorganisms isolated from sputum of suspected LRTI patients and susceptibility of the isolates toward different antibiotics. Culture positive rate was $29.93 \%$ in this study. A similar rate was shown by Oberoi A et al. (32\%), whereas a slightly higher rate was shown in the studies by Asati RK et al. (40.64\%), Edirisinghe LU et al. (56.56\%)..$^{4,10,11}$ In $70.07 \%$ no pathogenic organisms were reported; this might be due to reasons like prior use of antimicrobials, infection by atypical microorganisms, viral or fungal infections or some other conditions. And if we see the isolation rate in different age group there is no much significant differences. It was $33.33 \%$ in $<15$ years age group and similarly $25.49 \%$ in $15-40$ years, $31.93 \%$ in 41 60 years and $31.18 \%$ in $>60$ years age groups. In this study, the most common organisms are Klebsiella pneumoniae followed by Pseudomonas. Similar patterns of organisms were shown in the study done by Asati et al. ${ }^{4}$ Similarly study done by Edirishinghe LU et al. also showed Coliforms and Pseudomonas as the two most common organisms. ${ }^{11}$ But studies done by Oberoi $A$ et al. showed Streptococcus pneumoniae, Pseudomonas aeruginosa as the two most common organisms. ${ }^{10}$ The reason behind this may be Oberoi et al. did a study in community-acquired pneumonia and Streptococcus pneumoniae is the most common organism of community-acquired pneumonia. But in our study, only 23 Streptococcus pneumoniae were isolated. It may be due to this study is not only restricted to communityacquired pneumonia and another reason maybe most of the patients may have had antibiotics before coming to the hospital and Streptococcus pneumoniae being sensitive to those antibiotics.

In antimicrobial susceptibility testing, Vancomycin was observed to be $100 \%$ effective but this is only used for gram-positive bacteria but the most common organisms are gram-negative in case of RTIs. So Vancomycin cannot be recommended as empiric treatment in LRTIs but highly effective in case of Gram-positive bacteria. Similarly, in a study done by Oberoi et al, Vancomycin seemed to be $92.7 \%$ effective. ${ }^{10}$ Other effective antimicrobials shown to be Tobramycin $84.54 \%$, Imipenum $84.83 \%$, Amikacin 71.87\%, Gentamicin 70.97\%. Similarly, Asati RK reported Amikacin to be $92.7 \%$ effective and Gentamicin $41.3 \% .^{4}$ Other antimicrobials like Ceftazidime, Ciprofloxacin, Ceftriaxone, Cotrimoxazole seemed to be moderately effective having efficacy rate of $64.76 \%, 64.74 \%, 56.62 \%$ and $51.26 \%$ respectively. Most of the organisms isolated showed highly resistant to Amoxycillin-Clavulanic acid (92.85\%), Ampicillin $(84.1 \%)$, Cefazolin (82.47\%) and Ofloxacin (60.16\%).

Klebsiella, the most common isolate also showed high sensitivity to Imipenem (86.9\%), Amikacin (83.63\%) and Gentamicin (71.51\%) and high resistance to Ampicillin (97.36\%), Amoxycillin-clavulanic acid $(97.53 \% \%)$. Ciprofloxacin (71.6\%). Cotrimoxazole (58.14\%), Ceftriaxone (58.92\%), Cefotaxime $(52.89 \%)$ were moderately effective. A study done by Shilpa K, et al. showed $66.66 \%, 62.50 \%$ and $56.66 \%$ sensitivity towards Amikacin, Gentamicin, and Imipenum respectively. ${ }^{12}$ The second most common organism Pseudomonas, also an important causative agent for nosocomial infection, was found to be sensitive to Imipenem, Amikacin, Ciprofloxacin, Gentamicin. Cephalosporin group like Cefepime (57.14\%), Ceftazidime (53.92\%), Cefotaxime (41.17\%) were relatively less effective against Pseudomonas. Staphylococcus were highly resistant to Cephazoline (88.88\%), Cefotaxime (61.76\%), Ceftriaxone (50\%), Ceftazidime (100) whereas sensitive to Vancomycin (100\%), Amikacin (72.73\%), Imipenem(66.67\%) and Gentamicin(63.64\%). Streptococcus pneumoniae was highly sensitive to most of the antimicrobials used. $100 \%$ of isolated Streptococcus pneumoniae were sensitive to Cefotaxime, Ceftazidime, Ceftriaxone, Ofloxacin, Gentamicin. Efficacy of Ciprofloxacin was $95 \%$. In our region, most of the patients have the habit of coming to the hospital only after taking antibiotic and not being cured. This could be one of the reasons behind less number of Streptococcus pneumoniae isolation. Limitations of the study are: this is a retrospective study, only those antimicrobials which were tested were included, all the microorganisms were not tested against all the antimicrobials, only selected antimicrobials were tested during routine tests.

\section{CONCLUSIONS}

In case of suspected LRTIs, we have to think a lot to prescribe antimicrobials empirically. Proper diagnosis, identification of causative agents and their antimicrobial susceptibility pattern are important steps towards limiting the irrational use of antimicrobials. This study along with other related studies are sufficient to say that most of the isolated organisms are highly resistant to most of the antimicrobials. The types of bacteria isolated and their antimicrobial susceptibility pattern may be different from place to place and time to time.

\section{ACKNOWLEDGEMENTS}

We gratefully acknowledge all other faculty members and staff of the Department of Microbiology and all the staff of the medical record section of KIST Medical College for their kind support in carrying out this research.

Conflict of Interest: None. 


\section{REFERENCES}

1. Joseph P, Mizgerd SD. Acute Lower Respiratory Tract Infection. N Engl J Med. 2008;358(7):716-24. [PubMed | Full $\underline{\text { Text }} \mid \underline{\mathrm{DOI}}]$

2. Lim WS, Baudouin SV, George RC, Hill AT, Jamieson C, Le Jeune, et al. British Thoracic Society. Guidelines for the management of community-acquired pneumonia in adults. Thorax; 2009 Oct;64Suppl 3:iii1-55. [PubMed | Full Text | DOI]

3. Mandell LA, Wunderink RG, Anzueto A, Bartlett JG, Campbell GD, Dean NC, et al. Infectious Diseases Soci ety of America/American Thoracic Society consensus guid elines on the management of community-acquired pneumo nia in adults. Clin Infect Dis. 2007;44:S27-72. [ $\underline{\text { Text }}$ | DOI]

4. Asati RK. Antimicrobial Sensitivity Pattern of Klebsiella Pneumoniae isolated from Sputum from Tertiary Care Hospital, Surendranagar, Gujarat and Issues Related to the Rational Selection of Antimicrobials. Sch J App Med Sci. 2013;1(6):928-33. [Full Text]

5. Helms M, Vastrup P, Gerner-Smidt P, Molbak K. Excess mortality associated with antimicrobial drug resistant Salmonella typhimurium. Emerg Infect Dis. 2002 May;8(5):490-5. [PubMed | Full Text | DOI]
6. Travers K, Barza M. Morbidity of infections caused by antimicrobial-resistant bacteria. Clin Infect Dis. 2002 Jun;34(3):S131-4. [․ㅏbMed | Full Text | DOI]

7. Byarugaba DK. A view on antimicrobial resistance in developing countries and responsible risk factors. Int J Antimicrob Agents. 2004;24(2):105-10. [라Med | Full Text | DOI]

8. Betty AF, Daniel FS, Alice SW. Bailey and Scott's Diagnostic Microbiology. 12th ed. Philadelphia: Mosby; 2007. p. 216-47. [Full Text]

9. Matthew AW, Franklin RC, William AC, Michael. ND, George ME, David WH, et al. Performance standards for antimicrobial disc susceptibility test, Approved standard in Clinical and Laboratory Standards Institute (CLSI). M2-A9. 9th ed. Pennsylvania: Wayne; 2006. 26 p. [Full Text ]

10. Oberoi A, Aggarwal A. Bacteriological Profile, Serology and Antibiotic Sensitivity Pattern of Micro-organisms from Community Acquired Pneumonia. JK Science. 2006 April-June;8(2):79-82. [Full Text]

11. Edirisinghe LU, Kalukottage P, Maziama MNN, deSilva PV. A retrospective observational study on the sputum samples received by the Department of Microbiology, Teaching Hospital, Karapitiya in the year 2007. Galle Medical Journal. 2009 September;14(1):10-14. [Full Text]

12. Shilpa K, Thomas R, Ramyashree A. Isolation and Antimicrobial sensitivity pattern of Klebsiella pneumoniae from sputum samples in a tertiary care hospital. IJBAR. 2016;7(2):53-7. [Full Text | DOI] 